\title{
不同厚度四面体非晶碳薄膜的高通量制备及表征
}

\author{
魏 菁 ${ }^{1,2}$, 李汉超 ${ }^{1,3}$, 柯培玲 ${ }^{1}$, 汪爱英 ${ }^{1}$
}

(1. 中国科学院宁波材料技术与工程研究所, 中国科学院海洋新材料与应用技术重点实验室, 浙江省海洋材料与防护技术 重点实验室, 宁波 315201; 2. 中国科学院大学, 北京 100049; 3. 上海科技大学 物质科学与技术学院, 上海 201210)

摘 要: 材料基因组工程能大幅度提高材料研发速度, 降低材料研发成本, 近年来受到广泛关注。本研究采用高通 量制备工艺, 结合碳等离子体束流和基片位置的调控, 利用自主设计研制的 $45^{\circ}$ 双弯曲磁过滤阴极真空电弧设备, 沉积了厚度为 4.7 183 nm 的系列四面体非晶碳(ta-C)薄膜, 使用椭偏仪、原子力显微镜、拉曼光谱仪和 X 射线光电 子能谱仪(XPS)表征了厚度对 ta-C 薄膜表面粗䊅度、微结构和原子键态的影响。结果表明: 通过碳等离子体束流和 基片位置的调控，实现了不同厚度 ta-C 薄膜的高通量制备。尽管膜厚不同，所制备的 ta-C 薄膜均具有几乎不变的 光滑表面 $\left(R_{\mathrm{a}}=(0.38 \pm 0.02) \mathrm{nm}\right)$ 和色散值 $(\operatorname{Disp}(G))$, 说明不同厚度 ta-C 薄膜的 $\mathrm{sp}^{3}$ 含量、 $\mathrm{sp}^{2}$ 团簇尺寸保持相对稳定。 XPS 结果进一步证实 ta-C 薄膜的 $\mathrm{sp}^{3}$ 相对含量均维持在 $(55 \pm 5) \%$ 。此外, 不同厚度 ta-C 薄膜的光学带隙 $E_{\mathrm{opt}}$ 均保持 在(1.02 \pm 0.08$) \mathrm{eV}$ 。相关结果为设计制备结构和光学性能可控的不同厚度 ta-C 薄膜提供了一种新思路。

关 键 词: 四面体非晶碳膜; 高通量; 厚度; 微结构; 光学带隙

中图分类号: TQ127 文献标识码: A

\section{Characterization of Tetrahedral Amorphous Carbon Film with Various Thickness by High Through-put Method}

\author{
WEI Jing ${ }^{1,2}$, LI Han-Chao ${ }^{1,3}$, KE Pei-Ling ${ }^{1}$, WANG Ai-Ying ${ }^{1}$
}

(1. Zhejiang Key Laboratory of Marine Materials and Protective Technologies, Key Laboratory of Marine Materials and Related Technologies, Ningbo Institute of Materials Technology and Engineering, Chinese Academy of Sciences, Ningbo 315201, China; 2. University of Chinese Academy of Sciences, Beijing 100049, China; 3. Sohool of Physical Science and Technology, Shanghai Tech University, Shanghai 201210, China)

\begin{abstract}
Materials Genome Initiative (MGI), which greatly accelerates the research and development progress of new materials with reduced cost, has received widespread attention in recent years. In this report, high-quality ta-C films with different thicknesses, ranging from $4.7 \mathrm{~nm}$ to $183 \mathrm{~nm}$, were high through-put deposited by a home-built double $45^{\circ}$ bent filtered cathodic vacuum arc system, which was realized by the control of carbon plasma beam and substrate position. Meanwhile, the effects of film thickness on surface roughness, microstructure, and carbon atomic bond were investigated by atomic force microscope, spectroscopic ellipsometry, Raman spectra and X-ray photoelectron spectroscopy. Results show that the high through-put method by regulation of carbon plasma beam and selective placement of substrate enables to prepare ta-C films with various thicknesses. Particularly, the prepared ta-C films show almost constant smooth surface $\left(R_{\mathrm{a}}=(0.38 \pm 0.02) \mathrm{nm}\right)$ and the value of $\operatorname{Disp}(\mathrm{G})$ regardless of the thickness changes, revealing the unchanged size of $\mathrm{sp}^{2}$ cluster and $\mathrm{sp}^{3}$ content with different thicknesses. Furthermore XPS results confirm that $\mathrm{sp}^{3}$ relative content is kept at $(55 \pm 5) \%$. In addition, the optical band gaps of ta-C films with different
\end{abstract}

收稿日期：2018-01-16；收到修改稿日期：2018-03-09

基金项目：国家自然科学基金(51522106); 浙江省重点研发计划(2017C01001); 浙江省公益项目(2016C31121) National Natural Science Foundation of China (51522106); Zhejiang Key Research and Development Program (2017C01001); Public Projects of Zhejiang Province (2016C31121)

作者简介: 魏菁(1993-), 女, 博士研究生. E-mail: weijing@nimte.ac.cn

通讯作者：汪爱英，研究员. E-mail: aywang@nimte.ac.cn 
thicknesses remain at $(1.02 \pm 0.08) \mathrm{eV}$. These results could provide new insight into design and fabricate the controlled microstructure and optical property of ta-C film with different thicknesses.

Key words: tetrahedral amorphous carbon film; high through-put; thickness; microstructure; optical band gap

四面体非晶碳(Tetrahedral amorphous carbon, ta-C) 薄膜是一类不含氢, $\mathrm{sp}^{3}$ 含量高于 $50 \%$ 的类金刚石薄 膜 ${ }^{[1]}$ 。ta-C 薄膜集高硬度、低摩擦系数、耐磨损、 耐腐蚀、高化学稳定性和生物相容性等特性于一体, 在力学、摩擦学、生物学、电学、光学和热学等方 面展示出优良特性, 具有广泛的应用前景。但薄膜 内应力高、膜基结合力差, 导致制备 ta-C 厚膜非常 困难, 因此大部分研究只能制备数百纳米厚的本征 ta-C 薄膜 ${ }^{[2-4]}$ 。另外, 在纳米尺度下, ta-C 薄膜的密 度和成键特征会随厚度的变化发生显著变化, 这与 其内部 $\mathrm{sp}^{3}$ 含量等紧密相关。Palomäki 等 ${ }^{[5]}$ 发现当 ta-C 薄膜厚度在 7 100 nm 内增加时, 拉曼光谱拟合 得到的 $\mathrm{D}$ 峰与 $\mathrm{G}$ 峰的比值 $\left(I_{\mathrm{D}} / I_{\mathrm{G}}\right)$ 逐渐减小, $\mathrm{sp}^{3}$ 含量 逐渐增加; McCann 等 ${ }^{[6]}$ 也证实 ta-C 薄膜的厚度从 $30 \mathrm{~nm}$ 逐渐增加到 $100 \mathrm{~nm}, I_{\mathrm{D}} / I_{\mathrm{G}}$ 由 0.39 逐渐减小为 0.25 , 说明随着膜厚增加, $\mathrm{sp}^{2}$ 团簇尺寸减小, 推测 其 $\mathrm{sp}^{3}$ 含量逐渐增加。而 Sheeja 等 ${ }^{[7]}$ 制备的 ta-C 薄 膜, 膜厚在 0 80 nm 范围内增加时, $\mathrm{sp}^{3}$ 含量逐渐减 小; 膜厚超过 $80 \mathrm{~nm}$ 后先增加再保持相对稳定。 Zavaleyev 等 ${ }^{[8]}$ 在 ta-C 薄膜生长时, 通过控制相同的 祄底温度, 使不同厚度 $\mathrm{ta}-\mathrm{C}$ 薄膜的 $\mathrm{sp}^{3}$ 含量始终保 持在 $68 \%$ 左右。另一方面, 李晓伟等 ${ }^{[9]}$ 通过分子动 力学模拟得出超薄 ta-C 的密度和残余应力随着膜厚 的增加而减小, 这主要是由于膜厚的增加导致键 长、键角畸变的弛豫。许多研究也已表明膜厚是影 响 ta-C 薄膜微结构和机械性能的重要因素。随着膜 厚的增加, 薄膜具有更高的硬度和更好的承载能力, 因此耐磨性提高 ${ }^{[10]}$; 薄膜厚度减小, 抵抗外加载荷的 能力减弱, 会产生更多的缺陷 ${ }^{[11-12]}$ 。上述研究表明, ta-C 薄膜的厚度与其 $\mathrm{sp}^{2} / \mathrm{sp}^{3}$ 比例存在紧密相关性, 但是相关规律尚不明晰, 这对于 ta-C 薄膜, 尤其是 超薄 ta-C 薄膜的设计、制备和应用提出了诸多挑战。

磁过滤阴极真空电弧技术(FCVA)具有离化率 高的特点, 其磁过滤装置可以有效去除沉积束流中 的大颗粒, 有利于获得光滑致密的表面, 因此被广 泛用于沉积 ta-C 薄膜。本课题组前期对自主搭建的 FCVA 沉积系统进行了磁场的优化设计, 通过调控 基底负偏压、氩气流量、碳离子的入射角以及沉积 前的刻蚀工艺, 制备了高 $\mathrm{sp}^{3}$ 含量、低应力、致密无 通孔、表面光滑的高质量 ta-C 薄膜 ${ }^{[13-17]}$ 。另外, “材 料高通量制备”作为一种材料研发的新方法, 可以
在短时间内完成大量不同成分、不同化学计量比、 不同厚度样品的制备, 能够快速、大量、准确地获 得研究材料所需的关键数据, 有效加速材料的篮 选、优化及应用的进程，已成为国际新材料研发的 趋势 ${ }^{[18-19]}$ 。因此，结合 FCVA 技术的优势和材料“高 通量制备”的研发思路，可以快速制备不同厚度 ta-C 薄膜, 并阐明相关规律。

本工作利用自主研制的 $45^{\circ}$ 双弯曲 FCVA 设备, 通过等离子束流和沉积位置的有效调控, 实现了厚 度在 4.7 183 nm 范围的系列 ta-C 薄膜的高通量制备, 重点研究了厚度对 ta-C 薄膜微结构、表面形貌和光 学带隙的影响。

\section{1 实验方法}

\section{1 ta-C 薄膜的制备}

采用自主研制的 $45^{\circ}$ 双弯曲磁过滤阴极真空电 弧设备在 $\mathrm{p}(100$ )型单晶 $\mathrm{Si}$ 片(厚度 $450 \pm 50 \mu \mathrm{m}$ )基底 上沉积 ta-C 薄膜。

图 1(a)为 FCVA 设备的示意图, 主要包括电弧
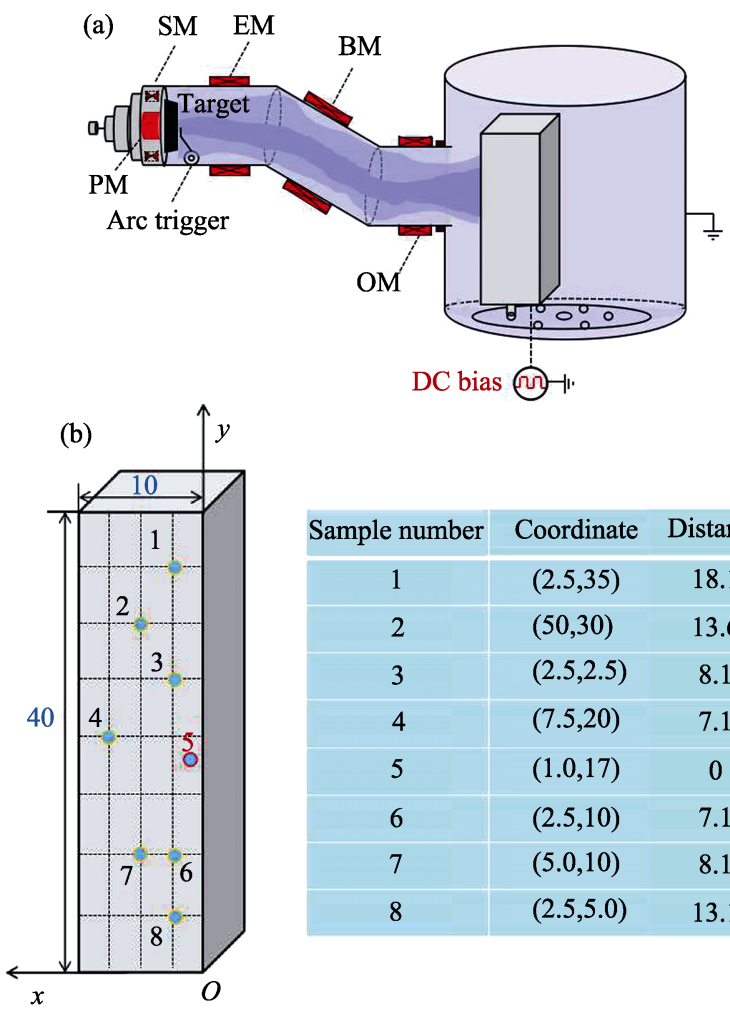

\begin{tabular}{|c|c|c|}
\hline Sample number & Coordinate & Distance \\
\hline 1 & $(2.5,35)$ & 18.1 \\
\hline 2 & $(50,30)$ & 13.6 \\
\hline 3 & $(2.5,2.5)$ & 8.1 \\
\hline 4 & $(7.5,20)$ & 7.1 \\
\hline 5 & $(1.0,17)$ & 0 \\
\hline 6 & $(2.5,10)$ & 7.1 \\
\hline 7 & $(5.0,10)$ & 8.1 \\
\hline 8 & $(2.5,5.0)$ & 13.1 \\
\hline
\end{tabular}

图 1 FCVA 设备示意图(a)和样品位置示意图(b)

Fig. 1 Schematic diagram of FCVA system (a) and sample position (b) 
表 1 ta-C 薄膜刻蚀和沉积的工艺参数

Table 1 Parameter of arc plasma etching and deposition for ta-C films

\begin{tabular}{cccccc}
\hline Process & Duct voltage/V & Arc current/A & Bias voltage $/ \mathrm{V}$ & Ar flux/sccm & Time/min \\
\hline Arc plasma etching & 10.0 & 50.0 & -300 & 20.0 & 5.0 \\
Film deposition & 10.0 & 50.0 & -80 & 3.0 & $15 / 30 / 45$ \\
\hline
\end{tabular}

蒸发源、45双弯曲磁过滤系统和安装有基体工件架 的真空沉积腔。靶材使用纯度为 $99.999 \%$ 、直径分 别为 $\phi_{\max }=70 \mathrm{~mm} 、 \phi_{\text {min }}=60 \mathrm{~mm}$ 的梯形柱状石墨靶。 其中, 由 $45^{\circ}$ 双弯曲的不锈钢弯管和永磁体(PM)、弧 源磁线圈 $(\mathrm{SM}) 、$ 拽引磁线圈 $(\mathrm{EM}) 、$ 弯曲磁线圈 $(\mathrm{BM})$ 和输出磁线圈 $(\mathrm{OM})$ 组成的磁过滤系统可以进行中 性大颗粒的有效过滤，设置 SM、EM、BM、OM 的 电流分别为 $5.0 、 4.5 、 4.5$ 和 $4.5 \mathrm{~A}$ 。为进一步提高 等离子体的传输效率，避免离化碳离子对弯管的轰 击, 对弯管施加 $10 \mathrm{~V}$ 的正电压。同时, 在磁过滤弯 管出口处安装有扫描线圈, 可调节等离子体束斑在 出口处进行扫描, 改变薄膜的沉积面积和均匀性。 真空沉积腔内有承载样品支架的转盘, 可自转和公 转。为改变沉积离子能量, 基体可通过偏压电源施 加一定的负偏压。

实验前尺寸为 $2 \mathrm{~cm} \times 2 \mathrm{~cm}$ 的 $\mathrm{Si}$ 基片用乙醇超声 清洗 $15 \mathrm{~min}$, 干燥后分别放置在四面体状基架的不 同区域。图 1(b)给出了样品位置的具体信息, 实验 中正对碳离子束流基架面的尺寸为： $40 \mathrm{~cm}($ 长) $\times$ $10 \mathrm{~cm}$ (宽), 根据碳离子束流的优化设计, 将不同位 置的样品标记为 $1 \sim 8$ 号, 通过建立直角坐标系, 可得 到不同编号样品的坐标。薄膜沉积过程中基架固定， 待设备本底真空抽至 $3.33 \times 10^{-3} \mathrm{~Pa}$, 先采用弧光等离 子体刻蚀对硅基片表面进行清洗, 然后沉积 ta-C 薄 膜，薄膜沉积时间分别为 $15 、 30$ 和 $45 \mathrm{~min}$, 具体的 工艺参数如表 1 所示。

\section{2 结构与成分表征}

采用光谱型椭偏仪(M-2000DI)测量薄膜厚度， 波长范围 190 1700 nm, 数据采用仪器自带的 WVASE32 软件进行分析; 使用扫描探针显微镜 SPM(Dimension 3100V)的接触模式表征样品表面的 粗粘度, $\mathrm{Si}_{3} \mathrm{~N}_{4}$ 探针的曲率半径为 $8 \mathrm{~nm}$, 测试面积为 $5 \mu \mathrm{m} \times 5 \mu \mathrm{m}$ 。通过显微激光共焦拉曼光谱仪(Renishaw inVia Reflex)表征薄膜的碳原子键结构, 为有效表 征 ta-C 薄膜中的 $\mathrm{sp}^{2} 、 \mathrm{sp}^{3}$ 杂化键, 测试激光波长选 用 $532 \mathrm{~nm}$ (可见)和 $325 \mathrm{~nm}$ (紫外), 其中 $\mathrm{sp}^{2}$ 杂化对应 $\pi$ 键的带隙为 $2.25 \mathrm{eV}$, 与 $532 \mathrm{~nm}$ 激光的能量相当; 而 $\mathrm{sp}^{3}$ 杂化对应 $\sigma$ 键的带隙为 $5.05 \mathrm{eV}$, 与 $325 \mathrm{~nm}$ 激 光的能量相当。采用 X 射线光电子能谱分析仪(AXIS UTLTRA DLD)分析薄膜的元素成分以及价态信息, 选用分辨率为 $0.48 \mathrm{eV}$ 的 $\mathrm{Al}$ 单色 $\mathrm{K} \alpha$ 射线源。

\section{2 结果与讨论}

\section{1 高通量制备不同厚度的 ta-C 薄膜}

为了同一批次获得不同厚度系列的样品，实验 调节了碳等离子体束流和基片的位置。采用相同的 工艺参数沉积 ta-C 薄膜, 不同样品的膜厚和沉积速 率随时间的变化如图 2 所示。

从图 2(a)中可以看出，当沉积时间分别为 15 、 30 和 $45 \mathrm{~min}$ 时, 5 号样品的膜厚均最大, 其余样品 的膜厚逐渐减小, 4 号、6 号和 3 号、7 号分别与 5 号样品呈中心对称。造成这种现象是由于在电弧沉 积过程中，虽然扫描线圈控制束流进行扫描，但四 面体基架不同区域的碳离子通量仍存在差异，因此 在不同位置的 ta-C 薄膜厚度不同。实验通过设定弯 管口的扫描参数，调控了碳等离子体束流的分布特 性，使碳等离子体的密度分布中心偏离到 5 号样品 位置处。进一步以 5 号样品为中心, 不同直径下可获 得不同厚度的等厚线。本实验中 4 号和 6 号样品对应 中心往外 $7.1 \mathrm{~cm}, 3$ 号和 7 号样品对应中心往外 $8.1 \mathrm{~cm}$, 2 号和 8 号对应中心往外 $13 \mathrm{~cm}$ 。
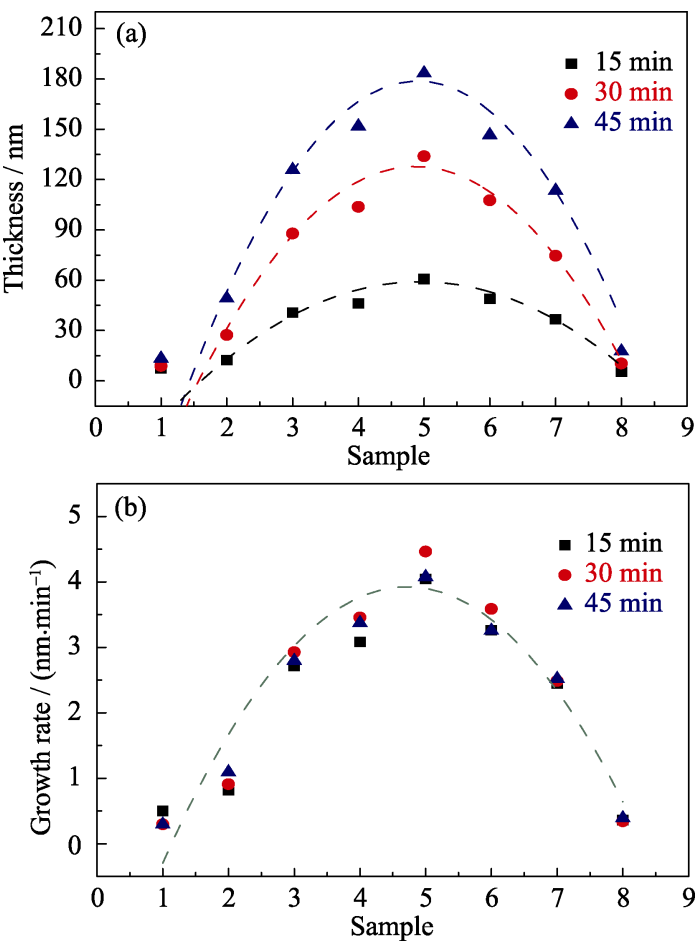

图 2 不同样品厚度和生长速率的变化(虚线便于观察规律)

Fig. 2 Thicknesses (a) and growth rates (b) changes of different samples (The dotted lines faciliate observation) 
而从图 2(b)中可以看出, 对于不同的样品, 改 变沉积时间获得的生长速率基本相同, 表明四面体 基架上不同位置处碳离子通量的差异相对稳定。因 此, 通过碳等离子体束流和基片位置的调节实验, 实现了系列不同厚度 ta-C 薄膜的高通量制备。

\section{2 厚度对表面粗龯度的影响}

图 3 为 ta-C 样品的表面粗糙度随厚度的变化曲 线。当膜厚由 $4.7 \mathrm{~nm}$ 增加到 $183 \mathrm{~nm}$ 时, ta-C 薄膜的 表面粗粘度 $R_{\mathrm{a}}$ 基本保持在 $(0.38 \pm 0.02 \mathrm{~nm})$, 而 $\mathrm{Si}$ 祄 底的表面粗粘度为 $0.45 \mathrm{~nm}$ 。沉积 ta-C 薄膜后, $\mathrm{Si}$ 祄底表面粗糙度降低, 表明沉积 ta-C 后有利于获得 光滑的表面。根据碳膜生长的分子动力学模拟, 波 谷处吸附位点的面积大于其他位置, 因此离子会优 先在波谷沉积 ${ }^{[20]}$ 。Zhong 等 ${ }^{[21]}$ 的实验结果表明当 $\mathrm{Si}$ 祄底的粗糙度为 $0.46 \mathrm{~nm}$ 时, 在沉积过程中碳离子 会在谷底择优生长, 导致表面平坦化。由此表明, 采 用 $45^{\circ}$ 双弯曲 FCVA 制备的 ta-C 薄膜具有填充基底 凹坑的作用，进而利于光滑表面。

\section{3 膜厚对 ta-C 薄膜原子键态的影响}

为进一步分析高通量制备的 ta-C 样品的膜厚与 微结构的关系，图 4(a) (c)分别给出了不同厚度 ta-C 薄膜的拉曼测试结果。图 4(a)为不同厚度 ta-C 薄膜 的紫外拉曼谱图, 可见在 $1100 \sim 1800 \mathrm{~cm}^{-1}$ 之间出现 了典型的非晶碳特征宽峰, 用 Gaussian 函数对宽峰 进行分峰拟合(其中厚度为 $4.7 \mathrm{~nm}$ 的 ta-C 薄膜, 拉 曼信号强度过低, 无法拟合)得到 $T$ 峰、 $D$ 峰、 $G$ 峰, 其中 $\mathrm{T}$ 峰对应 $\mathrm{C}-\mathrm{C} \mathrm{sp}$ 键的伸缩振动, $\mathrm{D}$ 峰和 $\mathrm{G}$ 峰分 别对应 $\mathrm{C}-\mathrm{C} \mathrm{sp}^{2}$ 键的呼吸振动和伸缩振动; 根据 $\mathrm{T}$ 峰/ $\mathrm{G}$ 峰的积分强度比 $I_{\mathrm{T}} / I_{\mathrm{G}}$ 值及 $\mathrm{T}$ 峰峰位可以获得薄膜 的碳键结构信息 ${ }^{[22]}$ 。

从图 4(b)中可以看出, 随着膜厚的改变, $I_{\mathrm{T}} / I_{\mathrm{G}}$ 值 呈弥散分布, 变化规律不明显。进一步与可见拉曼

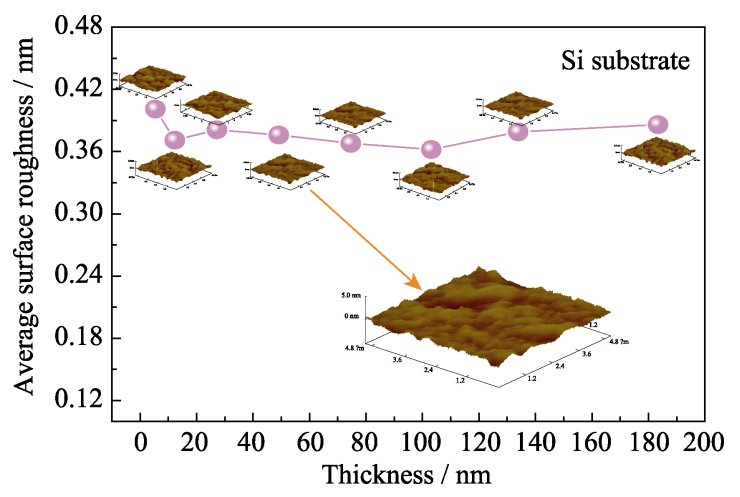

图 3 平均表面粗糙度随薄膜厚度的变化曲线

Fig. 3 Variations of the average surface roughness with film thickness
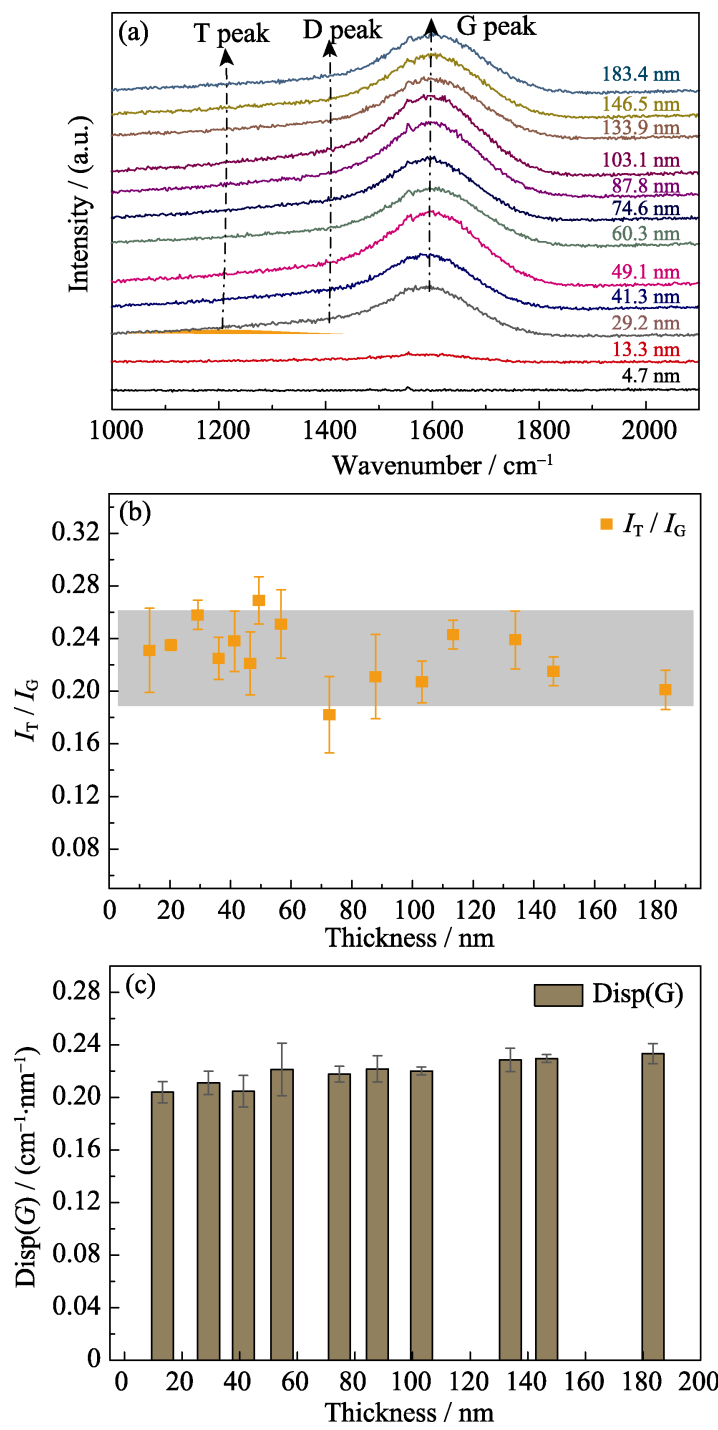

图 4 不同厚度 ta-C 薄膜的紫外拉曼谱图(a), $I_{\mathrm{T}} / I_{\mathrm{G}}(\mathrm{b})$, 色散 值(c)

Fig. 4 UV Raman spectra (a), $I_{\mathrm{T}} / I_{\mathrm{G}}(\mathrm{b}), \operatorname{Disp}(G)$ (c) of ta-C films with various film thickness.

(532 nm)和紫外拉曼 (325 nm)相结合, 根据式(1)计 算 ta-C 薄膜的色散值:

$$
\operatorname{Disp}(G)\left(\frac{\mathrm{cm}^{-1}}{\mathrm{~nm}}\right)=\frac{\operatorname{Pos}(G) @ 325-\operatorname{Pos}(G) @ 5332}{(532-325) \mathrm{nm}}
$$

图 4(c)中, 不同厚度的 ta-C 薄膜的色散值保持 相对稳定，表明薄膜的拓扑无序度维持不变，而拓 扑无序度和薄膜中 $\mathrm{sp}^{2}$ 团簇的尺寸、形状分布直接

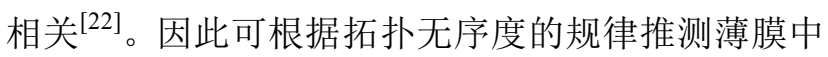
$\mathrm{sp}^{2}$ 团簇的尺寸基本不变, 进一步反映了 $\mathrm{sp}^{3}$ 杂化键 的含量基本不变。综上, 调控 $\mathrm{ta}-\mathrm{C}$ 膜厚, $\mathrm{sp}^{3}$ 杂化键 含量基本无变化, 同时 $\mathrm{sp}^{2}$ 团簇尺寸也基本不变。

为进一步精确表征不同厚度 ta-C 薄膜的 $\mathrm{sp}^{3}$ 含 量, 对 XPS 测试得到的 $\mathrm{C} 1 \mathrm{~s}$ 芯能级进行分峰拟合: $\mathrm{C}=\mathrm{C}\left(\mathrm{sp}^{2}\right)$ 和 $\mathrm{C}-\mathrm{C}\left(\mathrm{sp}^{3}\right)$ 采用 vogit 峰进行拟合, 峰位分 别位于 $(284.3 \pm 0.2) \mathrm{eV}$ 和(285.2 \pm 0.2$) \mathrm{eV}$; 在 $286.5 \mathrm{eV}$ 

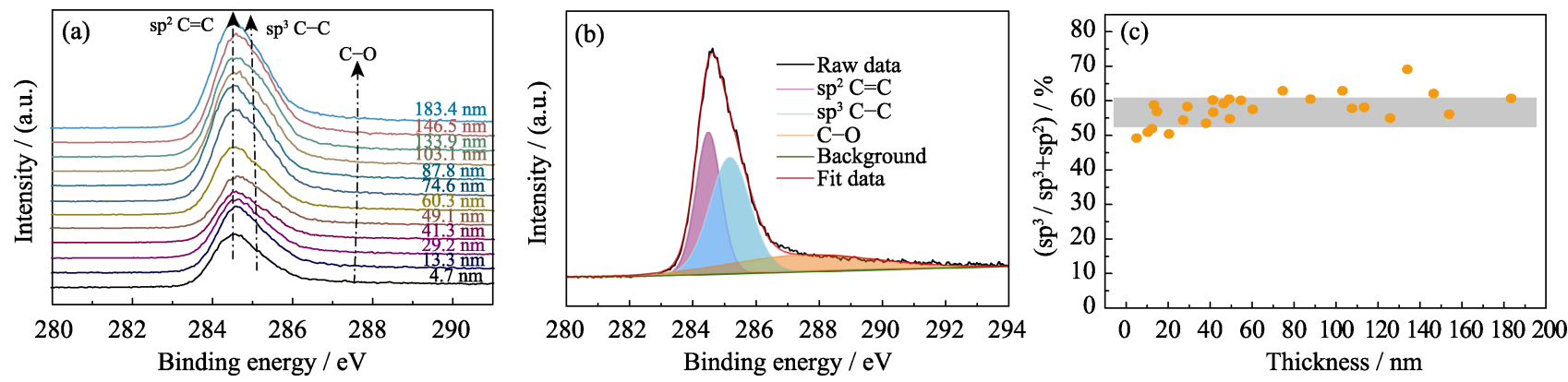

图 5 不同厚度 ta-C 薄膜的 XPS C1s 芯能级图谱(a)和 $\mathrm{sp}^{3}$ 含量 $(\mathrm{c})$, 典型 ta-C 薄膜 $(41.3 \mathrm{~nm}$ )的 $\mathrm{C} 1 \mathrm{~s}$ 芯能级谱线(b)

Fig. 5 XPS C1s core level spectra (a) and $\mathrm{sp}^{3}$ content (c) of ta-C films with various film thickness, typical C1s core level spectrum of ta-C film $(41.3 \mathrm{~nm})(\mathrm{b})$

附近还有 $\mathrm{C}-\mathrm{O}$ 结构(图 5(a))。根据分峰结果, 通过 公式(2)可以获得薄膜中 $\mathrm{sp}^{3} \mathrm{C}$ 的含量

$$
C_{\mathrm{sp}^{3}}=\frac{I_{\mathrm{sp}^{3}}}{I_{\mathrm{sp}^{3}}+I_{\mathrm{sp}^{2}}}
$$

其中, $I_{\mathrm{sp}^{3}}$ 和 $I_{\mathrm{sp}^{3}}$ 分别表示分峰拟合获得的 $\mathrm{sp}^{3}$ 峰和 $\mathrm{sp}^{2}$ 峰面积(图 5(b))。拟合结果如图 5(c)所示, 随着 薄膜厚度增加, $\mathrm{sp}^{3}$ 相对含量相对稳定, 保持在 $(55 \pm 5) \%$, 这与拉曼拟合结果一致。

\section{4 膜厚对光学带隙的影响}

根据 Robertson 提出的类金刚石薄膜的两相结 构模型，类金刚石薄膜可以描述为 $\mathrm{sp}^{2}$ 杂化的石墨 相团簇镶嵌在 $\mathrm{sp}^{3}$ 杂化的高度交联网络基质中 ${ }^{[1]}$ 。其 中, $\mathrm{sp}^{3}$ 杂化碳主要决定类金刚石薄膜的机械性能, 而 $\mathrm{sp}^{2}$ 杂化碳则主要决定类金刚石薄膜的光学、电 学及磁学性能。光学带隙是描述材料光学特性的重 要物理参量之一, 常用 Tauc 带隙计算定义类金刚石 薄膜的光学带隙。基于 ta-C 薄膜作为非晶半导体材 料, 可以根据 Tauc 公式计算不同厚度 ta-C 薄膜的光 学带隙 $E_{\mathrm{opt}}^{[1,23]}$

$$
\alpha h v=\mathrm{A}\left(h v-E_{\text {opt }}\right)^{2}
$$

将 $(\alpha h v)^{1 / 2}$ 对 $h v$ 作图, 对图中的直线部分进行线 性拟合得到截距即为光学带隙 $E_{\mathrm{opt}}$ 。而 ta-C 薄膜的 光学带隙 $E_{\mathrm{opt}}$ 主要取决于 $\mathrm{sp}^{2} \mathrm{C}$ 键的含量, $\mathrm{sp}^{2} \mathrm{C}$ 键的
含量增加会导致光学带隙减小 ${ }^{[24]}$ 。因此, 通过光学 带隙的变化可以验证 ta-C 薄膜 $\mathrm{sp}^{2} \mathrm{C}$ 键含量的变化。 采用椭偏仪测得薄膜的消光系数 $k$, 并计算其吸收 系数 $\alpha(\alpha=4 \pi k / \lambda)$ 。以 $40 \mathrm{~nm}$ 膜厚的 ta-C 为例, 消光 系数和光学带隙图谱分别如图 6(a) (b)所示。图 6(c) 为不同厚度 $\mathrm{ta}-\mathrm{C}$ 薄膜的光学带隙图, 可以看出不同 厚度 ta-C 薄膜的光学带隙 $E_{\text {opt }}$ 为 $(1.02 \pm 0.08) \mathrm{eV}$ 。整 体来看, $E_{\mathrm{opt}}$ 的大小与厚度变化无关, 验证了随着 ta-C 薄膜厚度的增加, $\mathrm{sp}^{3}$ 相对含量保持相对稳定。

\section{3 结论}

采用高通量方法, 调控碳离子束流在不同基片 位置的沉积, 实现了不同厚度 ta-C 薄膜的可控制备。

1)通过高通量的方法沉积 ta-C 薄膜, 薄膜的厚 度和生长速率与中心位置存在对称关系;

2)当膜厚由 $4.7 \mathrm{~nm}$ 增加到 $183 \mathrm{~nm}$ 时, ta-C 薄膜 的表面粗楉度基本保持在 $(0.38 \pm 0.02) \mathrm{nm}$, ta-C 沉积 后有利于获得光滑的表面;

3)拉曼、XPS 和光学带隙的结果综合表明, ta-C 薄膜厚度增加，薄膜中 $\mathrm{sp}^{3}$ 含量、 $\mathrm{sp}^{2}$ 团簇尺寸保持相 对稳定, 其中 XPS 得到的 $\mathrm{sp}^{3}$ 相对含量为 $(55 \pm 5) \%$, 计算获得的光学带隙为 $(1.02 \pm 0.08) \mathrm{eV}$ 。相关结果为 设计制备结构和光学性能可控的不同厚度 ta-C 薄膜 提供了一种新思路。
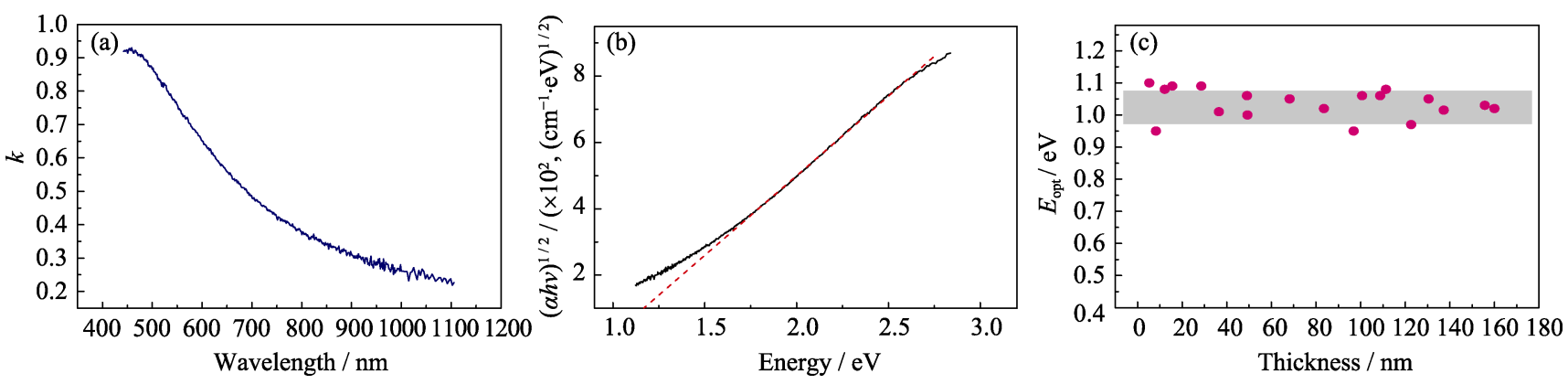

图 6 ta-C 薄膜典型的消光系数(a)和光学带隙(b)图谱(膜厚为 $41.3 \mathrm{~nm}$ ), 不同厚度 ta-C 薄膜的光学带隙图(c)

Fig. 6 Typical extinction coefficient (a) and optical bandgap (b) spectra of ta-C film with thickness of $41.3 \mathrm{~nm}$, optical band gap of ta-C with different film thicknesses (c) 


\section{参考文献:}

[1] ROBERTSON J. Diamond-like amorphous carbon. Materials Sci-

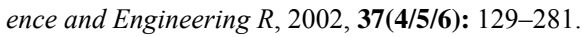

[2] ERDEMIR A, FENSKE G R, TERRY J, et al. Effect of source gas and deposition method on friction and wear performance of diamond-like carbon films. Surface and Coatings Technology, 1997, 94-95(1): 525-530.

[3] WANG M, JIANG X, STRIZKER B, et al. Adhesion of hydrogenated amorphous carbon films on silicon substrates and its enhancement. Thin Solid Films, 1991, 197(1/2): 57-66.

[4] ENKE K. Some new results on the fabrication of and the mechanical, electrical and optical properties of I-carbon layers. Thin Solid Films, 1981, 80(1/2/3): 227-234.

[5] PALOMAKI T, WESTER N, CARO M A, et al. Electron transport determines the electrochemical properties of tetrahedral amorphous carbon (ta-C) thin films. Electrochimica Acta, 2017, 225: 1-10.

[6] MCCANN R, ROY S S, PAPAKONSTANTINOU P, et al. The effect of thickness and arc current on the structural properties of FCVA synthesised ta-C and ta-C: N films. Diamond and related materials, 2005, 14(3): 983-988.

[7] SHEEJA D, TAY B K, LEONG K W, et al. Effect of film thickness on the stress and adhesion of diamond-like carbon coatings. Diamond and Related Materials, 2002, 11(9): 1643-1647.

[8] ZAVALEYEV V, WALKOWICZ J, KUZNETSOVA T, et al. The dependence of the structure and mechanical properties of thin ta-C coatings deposited using electromagnetic venetian blind plasma filter on their thickness. Thin Solid Films, 2017, 638: 153-158.

[9] LI X W, XU S P, KE P L, et al. Thickness dependence of properties and structure of ultrathin tetrahedral amorphous carbon films: a molecular dynamics simulation. Surface \& Coatings Technology, 2014, 258: 938-942.

[10] MIYAKE S, KUROSAKA W, OSHIMOTO K. Nanometre scale mechanical properties of extremely thin diamond-like carbon films. Tribology - Materials, Surfaces \& Interfaces, 2013, 3(4): 158-164.

[11] SUNDARARAJAN S, BHUSHAN B. Development of a continuous microscratch technique in an atomic force microscope and its application to study scratch resistance of ultrathin hard amorphous carbon coatings. Journal of Materials Research, 2001, 16(2): $437-445$.
[12] MA X G, KOMVOPOULOS K, WAN D, et al. Effects of film thickness and contact load on nanotribological properties of sputtered amorphous carbon thin films. Wear, 2003, 254(10): 10101018.

[13] LI H B, SUN L L, WU G S, et al. Simulation of magnetic field distribution in doubly-bent filter cathode of vacuum arc film growth setup. Chinese Journal of Vacuum Science and Technology, 2008, 29(3): 614-620.

[14] CAI J, YANG W, DAI W, et al. Effect of substrate bias on structure and properties of Ta-C films. China Surface Engineering, 2011, 24(6): 62-67.

[15] CAI J, YANG W, KE P L, et al. Influence of Ar flow rate on microstructures and tribological properties of tetrahedral amorphous carbon films. Chinese Journal of Vacuum Science and Technology, 2012, 32(8): 740-745.

[16] XU S P, LI X W, HUANG M D, et al. Stress reduction dependent on incident angles of carbon ions in ultrathin tetrahedral amorphous carbon films. Applied Physics Letters, 2014, 104(14): 141908-1-4.

[17] GUO T, ZUO X, GUO P, et al. Effect of etching process on growth and properties of tetrahedral amorphous carbon film. Surface Technology, 2017, 46(4): 143-149.

[18] ZHAO J C. A perspective on the materials genome initiative. Chinese Journal of Nature, 2014, 36(2): 89-104.

[19] WANG HAI-ZHOU, WANG HONG, DING HONG, et al. Progress in high-throughput materials synthesis and characterization. Science \& Technology Review, 2015, 33(10): 31-49.

[20] EVANS J W. Factors mediating smoothness in epitaxial thin-film growth. Physical Review B, 1991, 43(5): 3897-3905.

[21] ZHONG M, ZHANG C H, LUO J B. Effect of substrate morphology on the roughness evolution of ultrathin DLC films. Applied Surface Science, 2008, 254(21): 6742-6748.

[22] FERRARI A C, ROBERTSON J. Raman spectroscopy of amorphous, nanostructured, diamond-like carbon, and nanodiamond. Philosophical Transactions, 2004, 362(1824): 2477-2512.

[23] TAUC J, GRIGOROV R, VANCU A. Optical properties and electronic structure of amorphous germ anium. Phys. Status Solidi, 1966, 15(2): 627-637.

[24] 薛群基, 王立平. 类金刚石碳基薄膜材料. 北京: 科学出版社, 2012, 585 . 